\title{
Data-driven Modeling of Ship Motion Prediction Based on Support Vector Regression
}

\author{
Bikram Kawan $^{1}$ Hao Wang ${ }^{1}$ Guoyuan Li $^{2} \quad$ Khim Chhantyal $^{3}$ \\ ${ }^{1}$ Faculty of Engineering and Natural Sciences, Norwegian University of Science and Technology, Norway, \\ bikramkawan@gmail.com, hawa@ntnu. no \\ ${ }^{2}$ Faculty of Maritime Technology and Operations, Norwegian University of Science and Technology, Norway, \\ guoyuan.li@ntnu.no \\ ${ }^{3}$ Faculty of Technology, Natural Sciences, and Maritime Sciences, University College of Southeast Norway, Norway, \\ khim.chhantyal@usn.no
}

\begin{abstract}
This paper presents a flexible system structure to analyze and model for the potential use of huge ship sensor data to generate efficient ship motion prediction model. The noisy raw data is cleaned using noise reduction, resampling and data continuity techniques. For modeling, a flexible Support Vector Regression (SVR) is proposed to solve regression problem. In the data set, sensitivity analysis is performed to find the strength of input attributes for prediction target. The highly significant attributes are considered for input feature which are mapped into higher dimensional feature using non-linear function, thus SVR model for ship motion prediction is achieved. The prediction results for trajectory and pitch show that the proposed system structure is efficient for the prediction of different ship motion attributes.
\end{abstract}

Keywords: Ship Motion time series Prediction, Support Vector Regression

\section{Introduction}

The maritime industry is one of the key business backbones in Norway and has experienced significant increase in recent years. Ships are - driving force for maritime business and - important in aspect of companies and safety, (Baldauf et al., 2013). The dynamics of ships is complex due to control system forces and external forces, (Fossen, 2002; Sørensen, 2011). Hydrodynamic perturbations are also induced from ship motions. The external forces are a combination of wind, waves and sea currents. The control system perturbation is defined by propulsion and steering system of the ship, (Fossen, 2002). The ship motion is non-linear due to resultant forces obtained from the combination of external forces and control system. The priority of health, safety, environment and economic loss are in high priority for ship maneuvering. Therefore, a prediction model will be useful to take consideration of different factors in ship motion planning which is important for human safety, loss of economy and eventually to increase the efficiency of the ship.
There are different types of sensors installed in the ship. The information collected from sensors are used directly or indirectly. The real-time purpose is to maneuverer the ship and for control signals. The indirect use can be very useful for diagnose purpose in future to measure performance of different components of ships such as propeller blade, motor etc. The information produced from sensors are normally huge size which is in the form of big data, (Kaisler et al., 2013) for the several years' tenure. Big data are the huge source of information if we can dig into effectively. The information hidden are valuable for ship owners and companies for the prediction, identifying the patterns.

The accurate prediction of ship motion can be challenging due to high non-linearity of ship dynamics, the variable operational parameters of the vessel and the stochastic external excitations exerted by waves of the wind, (Pena et al., 2011). Many works are carried out for ship motion prediction. The use of traditional potential theory such as Kalman filter used in the frequency domain is not suitable due to the nonlinearity of ship dynamics and ocean for the estimation of actual ship behavior, (Blischke et al., 2011). Even though traditional algorithm may provide reasonable solutions, it gets complicated and requires more time to solve the problem mathematically, (Min-Seok, 2013). Classical model based approach lacks the generalization capabilities. Even though Extended Kalman filter can work in non-linearity to some extent but if failed when the non-linearity and complexity increases. SVR is one type of non-linear models which is good for solving a complex problem. Due to approximation ability, it solves models which are hard to solve using ordinary mathematical expression. Due to non-linearity behavior, there occurs some degree of uncertainty. Therefore, some intelligent techniques are required with the ability of generalization to solve the uncertainty in ship motion.

The successful manoeuvring operation of the ship is essential. Most important and key technique, such as information fusion, danger prediction technology traffic flow estimation, etc., cannot work without it. Therefore, the prediction of future time step for manoeuvring 
of the ship is useful to implement the control forces accurately. The research based on the application of artificial intelligence such as neural network, genetic algorithm, and Fuzzy Logic have shown the great potential for the prediction of ship behavior. A lot of interesting work has been carried out such as ship roll motion time series forecasting (Pena et al., 2011), online ship roll motion prediction (Yin et al., 2014), ship motion prediction (Zhao et al., 2004), ship trajectory based on backpropagation neural network (Xu et al., 2011) and ship path following (Xia et al., 2013). From (Luo and Cai, 2014; Ristic et al., 2008; Ma et al., 2003; Yin et al., 2013) it shows great potential to implement the SVR for the ship motion prediction.

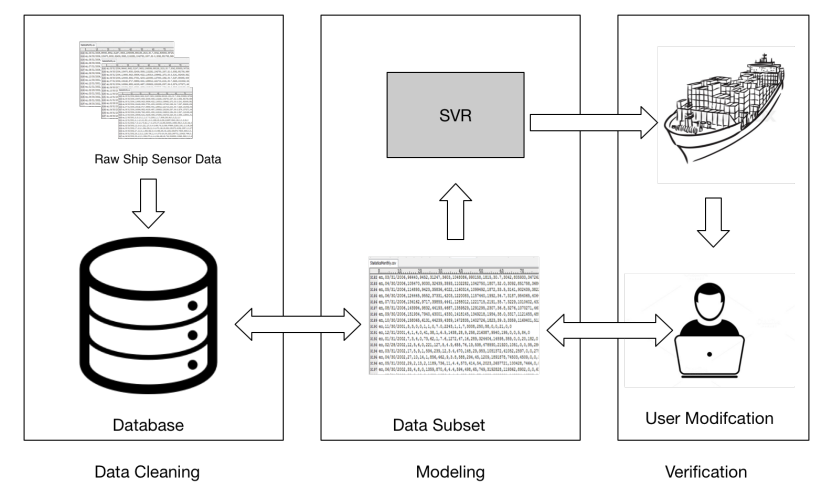

Figure 1. System structure of ship motion prediction. (Li et al., 2016)

\section{Methods}

The system structure for the prediction of ship motion will be based on the framework proposed as shown in Fig 1. The proposed framework consists three blocks. The first block of system structure is data cleaning. It is important to clean sensory noises and resampling the data if required to improve the model results. The second block i.e. Data analysis and modeling. Data analysis is carried out with the help of correlation analysis, which helps to identify the relation between different sensors attributes. The result obtained from correlation statistics is a guideline for the modeling of SVR. The third block is verification. The unique data which is not involved during learning is used for verification of our model. The user can import data from any ship model and make modification in the model to make the prediction better making our framework flexible.

\subsection{Data Collection}

The data is collected for three years from different sensors by our partner. The data module is divided into two category high frequency and low frequency. Each category has two subsets of data with frequencies from $1 \mathrm{~Hz}$ to $4000 \mathrm{~Hz}$. The high frequencies information is useful for the analysis of propulsion system using information obtained from vibration and torque sensors.
The information related ship environment is provided by low frequencies as shown in Table I. The intrinsic control parameters like rotational speed, thruster forces (from M2) induces the ship's extrinsic representation (M1). Our prediction model is based on low frequencies data (i.e. M1 and M2) only.

Table 1. Specification of Low Sampling Frequency Data.

\begin{tabular}{cccc}
\hline Module & Frequency $[\mathrm{Hz}]$ & Parameter & Unit \\
\hline & & Speed & {$[\mathrm{m} / \mathrm{s}]$} \\
& & Position & {$[\mathrm{m}, \mathrm{m}]$} \\
& & Heading & {$[\mathrm{deg}]$} \\
M1 & \multirow{3}{*}{1} & Roll & {$[\mathrm{deg}]$} \\
& & Pitch & {$[\mathrm{deg}]$} \\
& & Yaw Rate & {$[\mathrm{deg} / \mathrm{s}]$} \\
& & Roll Rate & {$[\mathrm{deg} / \mathrm{s}]$} \\
& & Pitch Rate & {$[\mathrm{deg} / \mathrm{s}]$} \\
\hline \multirow{3}{*}{ M2 } & \multirow{3}{*}{1.65} & Rotational Speed & {$[\mathrm{RPM}]$} \\
& & Drive of motor & {$[\mathrm{W}]$} \\
& & Propeller pitch & {$[\mathrm{deg}]$} \\
\hline
\end{tabular}

\subsection{Data Cleaning}

The raw data may contain noise, incomplete information and redundant information. So, it is necessary to perform data cleaning. In our case noise sources are internal or external. The internal noises can be corrected from statistical estimation. The external noises coming from sensor is unavoidable in most case. In our case, the major external noises are generated from cables and the coupling of electric and magnetic fields, the measured temperature is full of spikes. The natural way of eliminating the noise is to apply low pass filter and apply median filtering, (Liu, 2013). The raw data is not continuous due to several reasons such sensors are not actively running all the time, sensor is broken for some time. Due to this there are some gaps in recording data. In addition, there might be jumping of data for some parameter such as heading. The standard value is always within $\left[0^{\circ}, 360^{\circ}\right]$. The heading degree changes from 360 to 0 after making one complete cycle. To remove this kind of discontinuity an algorithm is used to correct the such parameter in order to make the data consistent, (Li et al., 2016).

\subsection{Sensitivity Analysis}

Sensitivity analysis is carried to find out the importance of input attribute for the contribution of output. It is not necessary that all the attributes have significant contribution for the prediction. To measure the strength of the attribute how much it is important for the contribution of output is calculated using correlation between the input attribute and the target attribute. (Hamby, 1994) 


\subsection{SVR Modeling}

Support Vector Machine (SVM) was developed by Vapnik (Vapnik, 2013) and is used in many applications due to significant results. SVM are a set of related supervised learning methods applied in different machine learning applications like classification and regressions (Scholkopf and Smola, 2005). The basic process is to map the original data input space into the higher dimensional feature through non-linear mapping functions (Scholkopf and Smola, 2005). We have implemented SVM to solve regression problem as Support Vector Regression in our work (Chhantyal et al., 2016).

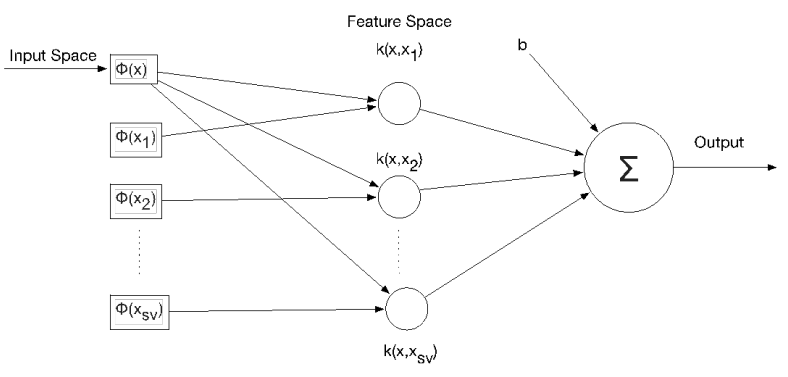

Figure 2. General architecture of SVR model. Input/output spaces consist of the variables shown in Table 1. (Chhantyal et al., 2016)

The linear regression model in feature space for SVR is represented as,

$$
y=\sum_{i=1}^{N_{s v}} w_{i} \Phi_{i}(x)+b
$$

where, $\Phi_{i}(x)$ is mapping function from input space to feature space and $N_{s v}$ is number of support vectors.

The performance of SVR model is measured by $\varepsilon$-insensitive loss function and defined as,

$$
L(d, y)=\left\{\begin{array}{ll}
|d-y|-\varepsilon & \text { for }|d-y| \geq \varepsilon \\
0 & \text { otherwise }
\end{array}\right\}
$$

The SVR approach is defined in (3) based on minimization of $\varepsilon$-insensitive loss and minimization of the norm of linear parameters $\|w\|^{2}$.

$$
\begin{gathered}
J=\frac{1}{2}\|w\|^{2}+C \sum_{i=1}^{N}\left|y_{i}-d_{i}\right|_{\varepsilon} \\
\text { subjected to }\left\{\begin{array}{l}
d_{i}-y_{i} \leq \varepsilon+\xi_{i} \\
y_{i}-d_{i} \leq \varepsilon+\xi_{i}^{*} \\
\xi_{i} \geq 0 \\
\xi_{i}^{*} \geq 0 \\
p
\end{array}\right\}
\end{gathered}
$$

where, C- regularization parameter, $\xi$ and $\xi^{*}$ are non-negative slack variables, which describe the loss function $\mathrm{J}$.

This primal optimization problem is then transformed into a dual problem (Chhantyal et al., 2016). The solution is defined in (4)

$$
y=\sum_{i=1}^{N_{s v}}\left(\alpha_{i}-\alpha_{i}^{*}\right) k\left(x_{i}, x\right)+b
$$

where, $\alpha$ and $\alpha^{*}$ are Lagrange multipliers and $k\left(x_{i}, x\right)$ is the kernel function used in mapping the input space to a higher dimensional feature space.

Radial Basis Function (RBF) kernel used in our work is defined as (5)

$$
k\left(x_{i}, x\right)=\exp \left(-\frac{\left\|x_{i}-x\right\|^{2}}{2 \sigma^{2}}\right)
$$

where, $\sigma$ is the width parameter of RBF kernel.

Support Vectors are those data within the training set, which are very close to the $\varepsilon$-insensitive tube. The number of support vectors is equal to the number of non-zero $\left(\alpha_{i}-\right.$ $\left.\alpha_{i}^{*}\right)$, which will determine the number of mapping vectors, determining the complexity of the model (Chhantyal et al., 2016). Therefore, the architecture of SVR depends on the number of support vectors as described in Fig. 2.

In this paper, the selection method for the parameters for the RBF and SVM is based on selecting $\mathrm{C}$ given by,

$$
C=\max \left(\left|\bar{y}+3 \sigma_{y}\right|,\left|\bar{y}-3 \sigma_{y}\right|\right)
$$

and the parameter $\varepsilon$ dependent on the level of noise in the training data is computed as,

$$
\begin{gathered}
\varepsilon=\tau \sigma_{n} \sqrt{\frac{\ln (n)}{n}} \\
\sigma_{n}=\left(\frac{k}{k-1} \frac{1}{n} \sum_{i=1}^{n}\left(y_{i}-\hat{y}_{i}\right)^{2}\right)^{1 / 2}
\end{gathered}
$$

For higher P-dimensional problems, $\mathrm{RBF}$ width parameter $\sigma$ can be set in the range $\sigma \approx(0.2 \text { to } 0.5)^{1 / P}$, which can be optimized further using train and error (Haykin, 2009).

\section{Experiment and Results}

The experiment is performed based on the raw data stored for three years to verify the system structure proposed in Section II. The case study aims to extract information from raw data and verify the SVR model for prediction of ship motions. This section describes the steps to develop our system to predict ship motion.

First, we import raw data in our database storage. For our case study, we are going to use a small subset for simplicity. Data cleaning is carried as the second step. We performed three data cleaning methods i) removing noise using low pass filter ii) resampling is performed to make both module (M1 and M2 shown in Table I) in same frequency iii) phase correction is performed to make the continuity of raw data. The detail process is discussed in our previous article (Li et al., 2016). The third step is 
to perform sensitivity analysis to find out the contribution of each attribute for the outputs as discussed in ( $\mathrm{Li}$ et al., 2016). For our case study, we found that surge velocity, sway velocity, yaw velocity, roll velocity, yaw velocity, position $\mathrm{x}$, position $\mathrm{y}$, heading, and roll has a significant relation for the prediction of the pitch.

The next step is to model SVR. Fig 2 shows how SVR model is constructed for our case. The selected variables are considered in the input feature space that are further transformed into high-dimensional space using radial basis function. Finally, the output is computed based on the local induced outputs from each hidden unit also.

The models are evaluated using Root Mean Square Error (RMSE) and Square Correlation Coefficient $\left(R^{2}\right)$. RMSE is a tool to measure a difference between the actual value and the value predicted by model defined in (9). The prediction results of the model are efficient, when RMSE value is near to 1 and the prediction results is treated bad when near to 0 .

$$
\operatorname{RMSE}(\hat{\theta})=\sqrt{E\left((\hat{\theta}-\theta)^{2}\right)}
$$

where, $\hat{\theta}$ is estimated value and $\theta$ is the actual value.

$R^{2}$ is used to measure the goodness of fit of a model and defined in (10). The estimate of the model is better if the value is closer to 1 .

$$
R^{2}=1-\frac{\sum_{i=1}^{n}\left(T_{i}-p_{i}\right)^{2}}{\sum_{i=1}^{n}\left(T_{i}-\bar{T}\right)^{2}}
$$

In this paper, we have only presented a case study for trajectory and pitch velocity. For both of our case studies, the size of the small subset of 2000 samples data was taken. The training size was 1750 and testing was 250 samples. The result obtained for trajectory is shown in Fig 3. The red line the actual trajectory while the blue one is trajectory predicted by SVR model.

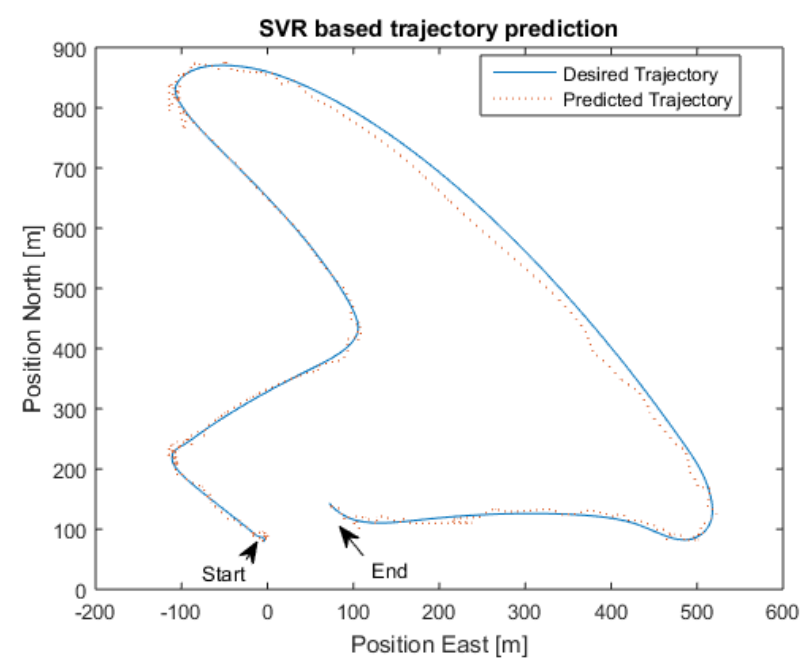

Figure 3. Prediction results of ship trajectory.
Fig 4 shows the prediction of the pitch. The red line is the desired pitch, and the blue line is the prediction obtained from SVR model.

In both of the case, it is clear from the Fig (3) and Fig (4) that the prediction from our model is good enough to predict trajectory and pitch. In addition, in both cases, $R^{2}$ is near to 1 and RMSE is around 0.1. This verifies that our model is good enough for the implementation.

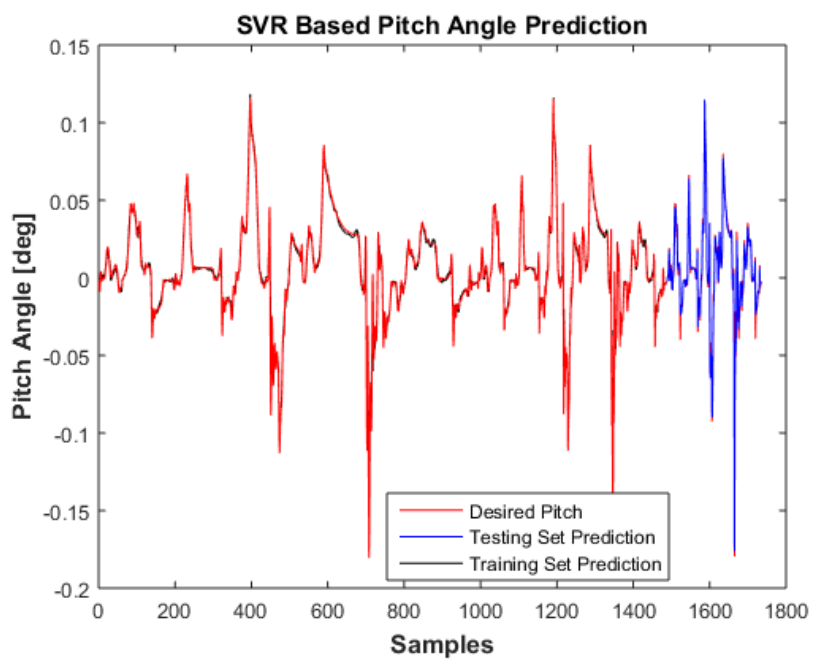

Figure 4. Prediction results of ship pitch.

\section{Conclusion}

In this paper, system structure for the prediction of ship motion is proposed from importing raw data to the verification of SVR model. First, the raw sensor data is cleaned before modeling. Then sensitivity analysis is performed to find the better relation between inputs to targets. Then flexible SVR model is constructed from which user can define the desired data set and user define model parameters. By applying training data set, SVR prediction model is obtained. As a final step, testing data is used for model verification. Two case studies for trajectory and pitch are carried out, and the results indicate that the proposed system outperformed for the ship motion prediction. In both case studies, RMSE is very small, and $R^{2}$ is close to 1 .

Future work will focus on the online prediction of ship motion which will be helpful for real-time prediction. In addition, the multi-step prediction will be studied for the longer time steps prediction that is essential for real world ship motion.

\section{Acknowledgement}

The authors would like to thank Mechatronics Lab, Faculty of Maritime Technology and Operations, NTNU i Ålesund for providing the data set for the research. 


\section{References}

M Baldauf, R Baumler, A Olcer, T Nakazawa, K Benedict, and $M$ Fischer, Sand Schaub. Energy-efficient ship operation training requirements and challenges. TransNav: International Journal on Marine Navigation and Safety of Sea Transportation, 7(2), 2013.

W R Blischke, M R Karim, and D P Murthy. Reliability. Technical report, Warranty Data Collection and Analysis, 2011.

K Chhantyal, H Viumdal, S Mylvaganam, and G Elseth. Ultrasonic level sensors for flowmetering of non-newtonian fluids in open venturi channels: Using data fusion based on artificial neural network and support vector machines. In Sensors Applications Symposium (SAS), 2016 IEEE, 2016.

T I Fossen. Marine control systems: guidance, navigation and control of ships, rigs and underwater vehicles. Technical report, Marine Cybernetics, 2002.

D M Hamby. A review of techniques for parameter sensitivity analysis of environmental models. Environmental monitoring and assessment, 32(2):135-154, 1994.

S Haykin. Neural networks and learning machines. Pearson, Upper Saddle River, NJ, USA, 2009.

S Kaisler, F Armour, J A Espinosa, and W Money. Big data: Issues and challenges moving forward. In System Sciences (HICSS), 2013 46th Hawaii International Conference, 2013.

G Li, H Zhang, B Kawan, H Wang, O L Osen, and A Styve. Analysis and modeling of sensor data for ship motion prediction. In OCEANS 2016-Shanghai, IEEE, 2016.

Y Liu. Noise reduction by vector median filtering. Geophysics, 78(3):V79-V87, 2013.

W Luo and W Cai. Modeling of ship manoeuvring motion using optimized support vector machines. In Intelligent Control and Information Processing (ICICIP), 2014 Fifth International Conference, 2014.

$\mathrm{J}$ Ma, J Theiler, and S Perkins. Accurate on-line support vector regression. Neural computation, 15(11):2683-2703, 2003.

Kang Min-Seok. Ship motion prediction system development based on neural network. Technical report, 2013.

F L Pena, M Gonzalez, V D Casas, and R J Duro. Ship roll motion time series forecasting using neural networks. In Computational Intelligence for Measurement Systems and Applications (CIMSA), 2011 IEEE International Conference, 2011.

B Ristic, B La Scala, M Morelande, and N Gordon. Statistical analysis of motion patterns in ais data: Anomaly detection and motion prediction. In Information fusion, 2008 11th international conference, 2008.

B Scholkopf and A Smola. Support vector machines. Technical report, Encyclopedia of Biostatistics, 2005.

A J Sørensen. A survey of dynamic positioning control systems. Annual reviews in control, 35(1):123-136, 2011.
V Vapnik. The nature of statistical learning theory. Technical report, Springer science \& business media, 2013.

G Xia, J Liu, and H Wu. Neural network based nonlinear model predictive control for ship path following. In Natural Computation (ICNC), 2013 Ninth International Conference, 2013.

$\mathrm{T} \mathrm{Xu}, \mathrm{X} \mathrm{Liu}$, and X Yang. Ship trajectory online prediction based on bp neural network algorithm. In Information Technology, Computer Engineering and Management Sciences (ICM), 2011 International Conference, 2011.

J C Yin, Z J Zou, and F Xu. On-line prediction of ship roll motion during maneuvering using sequential learning rbf neuralnetworks. Ocean engineering, 61:139-147, 2013.

J C Yin, Z J Zou, F Xu, and N N Wang. Online ship roll motion prediction based on grey sequential extreme learning machine. Neurocomputing, 129:168-174, 2014.

$\mathrm{X}$ Zhao, R Xu, and C Kwan. Ship-motion prediction: algorithms and simulation results. In Acoustics, Speech, and Signal Processing, Proceedings (ICASSP'04) IEEE International Conference, 2004. 